\title{
Die digitalisierte Demokratie. Ein Überblick
}

\author{
Isabelle Borucki • Dennis Michels • Stefan Marschall
}

Online publiziert: 6. August 2020

(C) Der/die Autor(en) 2020

Zusammenfassung Digitalisierung und Demokratie stehen in einem spezifischen Wechselverhältnis zueinander. Der Beitrag beleuchtet die Entwicklung der theoretischen Perspektiven, die den Zusammenhang von Digitalisierung und Demokratie in der Formationsphase der digitalisierten Demokratie begleiten. Neben einer ,netzrealistischen Perspektive“ sticht dabei vor allem die Betrachtung von Digitalisierung und Demokratie als ko-evolutionärer Prozess auf dem Weg zu einer neuen Ordnung hervor. Zudem werden die zwei weiteren im Heft adressierten Bereiche der digitalen Öffentlichkeiten und digitalen Partizipation vorgestellt und ein Überblick über die Beiträge des Heftes gegeben.

\section{The digitalized democracy. An overview}

Abstract Digitalization and democracy are marked by a specific interdependence. The article sheds light on the evolution of theoretical perspectives that accompany the interrelation between digitalization and democracy in the forming phase of digitalized democracy. Two perspectives, the "net-realistic" perspective and the coevolutionary perspective, which sheds light on the interactive relationship of the two phenomena on their way to a new order, are underlined as the most fruitful approaches in contemporary analyses. Furthermore, the two other areas of the issue, digital publics and digital participation are introduced and an overview of the single contributions to this issue is given.

I. Borucki $(\square)$

Institut für Politikwissenschaft, NRW School of Governance, Universität Duisburg-Essen,

Lotharstraße 53, 47057 Duisburg, Deutschland

E-Mail: isabelle.borucki@uni-due.de 
„Digitalisierung“ ist das Buzzword der letzten Jahre schlechthin. Kaum eine Veranstaltung oder Publikation zum Thema Digitalisierung ist ohne den obligatorischen Hinweis vorstellbar, dass die Digitalisierung mittlerweile omnipräsent sei. Diese gefühlte Omnipräsenz wurde durch die Corona-Pandemie nochmals verstärkt, als Home-Office, Home-Schooling und Home-Networking per Videokonferenzen während des Lockdowns über Nacht zum Allgemeingut wurden. Gut, wer da entsprechend ausgerüstet war - sowohl mit dem entsprechenden Equipment als auch einem Home-Office-fähigen Beruf. Sichtbar wurden dabei auch längst bestehende digitale Ungleichheiten (Brake 2014; Wei 2012; Min 2010). Das erinnert daran, dass Digitalisierung und Digitalität als qualitativ transformative und insofern performative Prozesse bereits seit mehr als fünf Dekaden auf das gesellschaftspolitische Miteinander einwirken - und letzten Endes auch auf die Demokratie.

\section{Digitalisierung und Demokratie}

Der Zusammenhang von Digitalisierung und Demokratie ist heute in einer Phase, die als Formationsphase hin zu einer digitalisierten Demokratie gelten kann und ist weiterhin Gegenstand akademischer und gesellschaftlicher Diskussionen. Bis vor wenigen Jahren dominierte ein Verständnis, wonach das Internet als eine Art Naturphänomen über die gesellschaftlichen und politischen Systeme der Welt geradezu hereinzubrechen schien, was zunächst zur Frage führte, ob diese Entwicklung positive oder negative Effekte auf die demokratischen Systeme westlicher Gesellschaften haben würde (Bieber 2001; Kneuer 2013; Marschall 1998; Weiß 1998). Die Erwartungshaltung oszillierte dabei zwischen utopischen Visionen eines technologiegestützten Auswegs aus der Krise der repräsentativen Demokratie (Rheingold 1993) und dystopischen Szenarien eines Online-Überwachungsstaats (Dahlberg 2011 für eine Übersicht; Miller und Vaccari 2020). Zwischen den Polen fand sich zudem ein Plädoyer für eine ,netzrealistische Betrachtung“ (Kneuer 2013, S. 8), d.h. einer Differenzierung der Folgen der Digitalisierung in Bezug auf kleinteiligere Untersuchungsgegenstände, um in der Gesamtschau eine bessere Gegenüberstellung positiver und negativer Effekte hinsichtlich ihrer Wirkungsperspektive zu erzielen.

Obwohl Studien unter dieser Prämisse wertvolle Erkenntnisse lieferten und auch weiterhin erbringen, wächst ein Unbehagen über die unidirektionale Perspektive eines Einflusses „,des Internets“ auf „die Demokratie“. Aktuelle Reflektionen kreisen daher um die Idee, dass das Internet, das Web 2.0, Algorithmen und andere digitale Anwendungen durch menschliche Entscheidungen hervorgebrachte Ausprägungen digitaler Technologie darstellen und daher nicht deterministisch verstanden werden sollten (Hofmann 2019; Helbing 2019; Schröder und Schwanebeck 2017). Statt eines neutralen Übertragungskanals bildeten die spezifischen Anwendungen, in Form neuer sozialer Medien, Möglichkeitsräume für unterschiedliche Handlungsoptionen im demokratischen System. Politisches Handeln und Kommunikationsmedien beeinflussen sich wechselseitig. Ihre gegenwärtige Form ist dabei Ausprägung vergangener Entwicklungsentscheidungen und Nutzungserfahrungen sowie umgekehrt. So wirken aktuelle Anwendungen digitaler Technologie durchaus auf die Entwick- 
lung der Demokratie, wie es seit vielen Jahren untersucht wird; zugleich prägen allerdings gesellschaftspolitische Entwicklungen die weitere Richtung eines Formwandels der Digitalisierung, besonders sichtbar in neuen Kommunikationsmedien. Die sich herausbildenden Formen dieses ko-evolutionären Entwicklungsprozesses von Demokratie und Digitalisierung sind derzeit erst in Ansätzen erkennbar (Thaa und Volk 2018).

Dieses Heft der Zeitschrift für Politikwissenschaft erkundet die verschiedenen Bereiche des demokratischen Formwandels angesichts spezifischer Nutzungsweisen digitaler Technologie. Statt eines umfassenden und systematischen Aufarbeitens ${ }^{1}$ werden Schlaglichter auf verschiedene Phänomene geworfen, welche sich in der Gesamtschau zu einer vorläufigen Zustandsbeschreibung der digitalisierten Demokratie zusammenfügen. Gemeinsam ist den Beiträgen des Bandes die Suche nach Mustern in den sozialen Interaktionen, die auf Datafizierung, Vernetzung und Algorithmisierung in digitalen Anwendungen aufbauen. Dass dabei auch, aber nicht nur, die bekannten sozialen Medien Facebook und Twitter im Fokus stehen, verdeutlicht die angesprochene Bandbreite digitaler Möglichkeiten, die eine gegenwärtige, aber keinesfalls endgültige Form digitaler Kommunikations- und Beteiligungsmedien repräsentieren.

Im Fokus stehen einerseits digitalisierte Öffentlichkeiten, die, anders als etablierte Massenmedien, von einem aktiven, produktiven, vernetzten und permanent sendenden Publikum geprägt sind (Dohle et al. 2014; Henn et al. 2016; Hepp et al. 2018). In sozialen Medien wird Kommunikation räumlich und zeitlich entgrenzt, situativ und ständig vervielfältigt - und das bei einer Permanenz der produzierten Daten. Für eine Mediendemokratie bedeutet das eine Herausforderung bezüglich der politischen Willensbildung im herkömmlichen Sinne, da Populismus, Falschinformationen, Hass und Hetze sich schnell ausbreiten können (Tucker et al. 2017). Dies setzt Regierungen, Parteien, Medien und gesellschaftliche AkteurInnen gleichermaßen unter Druck, da politische Kommunikation selbst Teil der Politik ist (Saxer 1998, S. 25; Geisler und Sarcinelli 2002, S. 62; Korte und Fröhlich 2009, S. 101,), was insbesondere in Wahlkampfzeiten, den „Hochzeiten politischer Kommunikation" (Korte et al. 2018, S. 194), deutlich wird.

Andererseits wird ein Blick auf aktuelle Entwicklungen im Bereich der digitalen Partizipation geworfen. Hier treffen Anwendungen digitalisierter Kommunikation und bekannte Strukturen politischer Partizipation aufeinander (Kersting 2019). Neben digitalen Formen von Wahlentscheidungen über Personen und Sachfragen geht es dabei auch um deliberative und unverbindliche Formen der digitalen Partizipation, bei denen BürgerInnen Fragen gemeinsam diskutieren und nur teilweise anschließend darüber entscheiden. Demokratietheoretisch ist die kritische Hinterfragung angebracht, inwiefern politische Institutionen digitale kommunikative Beteiligung ohne Entscheidungsrecht als postdemokratische Reaktion auf Massenkommunikation und Beteiligungsforderung in Stellung bringen (Stalder 2016, S. 206; Crouch 2008). Anwendungsfälle finden sich insbesondere auf der lokalen Ebene, z. B. bei

\footnotetext{
1 Den gesellschaftlichen und politischen Wandel im Zeitalter der Digitalisierung beschreiben aus politikwissenschaftlicher Sicht Hofmann et al. (2019), in soziologischer Perspektive Stalder (2016), Nassehi (2019) und Baecker (2018).
} 
der Planung kommunaler Infrastrukturprojekte. Von deliberativer Beteiligung unterschieden werden sollte allerdings die demonstrative Partizipation, die von Seiten der Beteiligten größtenteils auf Expressivität und nur manchmal auf Deliberation ausgerichtet ist (Kersting 2019, S. 111). Äußerungen in sozialen Medien können je nach Betrachtung deliberativen oder expressiven Charakter tragen, ähnlich wie es von Parlamentsdebatten (deliberativ) oder Demonstrationen (expressiv) bekannt ist: Nicht alle Äußerungen zielen auf argumentativen Austausch. Forschungsleitende Fragen werden in Bezug auf Anwendungsfälle digitaler Partizipation in Richtung von Zielen, Effizienz und Effektivität, Inklusivität oder Gründen für spezifische Nutzungsmuster gestellt.

Die zusammenführende Perspektive aus Betrachtungen von digitalen Anwendungen, welche von Behörden oder politischen Organisationen bereitgestellt werden, sowie Art und Umfang der Nutzung dieser Anwendungen bergen Erkenntnisse über möglicherweise darin angelegte Ungleichheiten. Somit ist die Forschung zu digitaler Partizipation grundlegend für eine normativ geleitete Weiterentwicklung digitaler Anwendungen, welche die digitalen Spaltungen in der partizipativen Demokratie verringern oder vermeiden soll.

\section{Die Beiträge}

Zum Auftakt des Bandes wird ein theoretisch-konzeptioneller Blick auf den Zusammenhang von Digitalisierung und Demokratie geworfen. Im ersten Beitrag erarbeiten Sebastian Berg, Niklas Rakowski und Thorsten Thiel ein politikwissenschaftliches Verständnis von Digitalisierung auf Basis der Wechselwirkung technischer und gesellschaftlicher Entwicklungen. Nach einer Rekonstruktion von Digitalisierungsverständnissen aus den Nachbardisziplinen der Soziologie und der Rechtswissenschaft (Baecker, Nassehi, Reckwitz, Stalder und Vesting) schlagen sie eine Perspektive des Formwandels der Demokratie in der digitalen Konstellation vor, deren Konzeptualisierung von Politik und Demokratie über die der Nachbardisziplinen hinausgeht. Exemplifiziert wird ihre theoretische Perspektive am Wandel der Repräsentation in der digitalen Konstellation.

Anschließend steht das Thema digitale Öffentlichkeiten im Fokus der folgenden drei Beiträge. Pablo Joost, Marc Ziegele und Teresa K. Naab widmen sich im zweiten Beitrag des Bandes dem Diskussionsklima auf Facebook. Sie untersuchen begünstigende und hemmende Bedingungen für eine digitale Zivilcourage, d.h. ein Einschreiten in Online-Diskussionen, die von Aggression, Drohungen, Beleidigungen oder Abwertungen geprägt sind. Ihr Fallbeispiel der Gruppe \#ichbinhier zeigt unterschiedliche Bedingungen für ein Einschreiten mittels eigener Kommentare im Gegensatz zum Einschreiten durch Bewertung bereits vorhandener Kommentare. Eine politisch geprägte Nutzung der Plattform, ein Gefühl persönlicher Verantwortung und eine Erwartung, dass das Diskussionsklima sich nach dem Einschreiten verbessere, bilden hingegen die gemeinsame Grundlage der digitalen Zivilcourage.

Auch der dritte Beitrag beschäftigt sich mit der Kommunikation der NutzerInnen auf Facebook und betrachtet dabei den Austausch mit den politischen Parteien. Elina Spieß, Dennis Frieß und Anne Schulz widmen sich dem Phänomen des Popu- 
lismus, der durch die spezifischen Strukturen sozialer Medien zu florieren scheint. Ihre explorative Untersuchung der Facebook-Seiten von AfD, CDU und SPD bringt dabei erstmals den Populismusgehalt von Parteikommunikation mit dem Populismusgehalt der NutzerInnenkommentare unter den Posts der Parteien in Verbindung. Während Initialposts der AfD den höchsten Populismusgehalt aufweisen, sind es bei den Kommentaren die NutzerInnen der CDU. Bei beiden Parteien wird zudem nachgewiesen, dass von Populismus geprägte Kommunikation der Parteien ebenfalls populistische Anschlusskommunikation nach sich zieht, was für die SPD allerdings nicht festgestellt werden konnte.

Anschließend wird im vierten Beitrag, ebenfalls zum Thema digitale Öffentlichkeiten, der Fokus auf die Rolle des Journalismus in sozialen Medien gelenkt. Christian Nuernbergk untersucht die Gruppe der NutzerInnen von Twitter, die mit PolitikjournalistInnen in direkten Kontakt treten, indem er fragt, wer den Kontakt zu PolitikjournalistInnen sucht und mit welcher Motivation dies geschieht. Er zeigt auf, dass es den Twitter-NutzerInnen vorrangig um Expressivität geht, teilweise aber auch um deliberative Motive, da auch das Weitergeben von Feedback intendiert wird.

Die folgenden drei Beiträge behandeln das Thema digitale Partizipation. Zunächst werfen Bastian Rottinghaus und Tobias Escher im fünften Beitrag des Heftes einen Blick auf digitale BürgerInnenbeteiligungsverfahren zur Fahrradinfrastruktur in den drei nordrhein-westfälischen Städten Köln, Bonn und Moers. In ihrem Beitrag spüren sie den Bedingungen nach, die eine politische Beteiligung über digitale Instrumente begünstigen oder erschweren und suchen nach Maßnahmen, die eine gleichere Beteiligung fördern könnten. Ihre Ergebnisse bestätigen frühere Erkenntnisse über eine Verzerrung der Beteiligung in Richtung männlicher Bürger mittleren Alters mit überdurchschnittlicher Bildung, überdurchschnittlichem Einkommen sowie einem ausgeprägten Gefühl von Selbstwirksamkeit. Demgegenüber zeigen sie, dass die Informationen über den Beteiligungsprozess in den von ihnen untersuchten Fällen zwar gering, dafür aber gleich verteilt war. Was die Menschen allgemein zur Beteiligung in den untersuchten Verfahren anregte, war die eigene Betroffenheit, d.h. in diesem Fall sowohl die Unzufriedenheit mit der gegenwärtigen Fahrradinfrastruktur, als auch das Interesse an Verbesserungen, da man die Infrastruktur selbst zu nutzen beabsichtigt. Der Umstand, dass das Verfahren digital organisiert wurde, war einigen Menschen ein Beteiligungshindernis.

Im sechsten Beitrag des Heftes untersuchen Julia Schwanholz und Lavinia Zinser die Angebotsseite digitaler Beteiligung. Sie vergleichen sechs kommunale Plattformen der digitalen BürgerInnenbeteiligung, von denen drei über die Software Adhocracy, die anderen drei über die Software LiquidFeedback umgesetzt wurden. Ihre angebotsseitige Evaluation von Kriterien wie Zugang, Freiheit der Themensetzung, Ausrichtung auf das Gemeinwohl, Interaktivität, Transparenz und Responsivität zeigen, dass Plattformen auf Basis der Software Adhocracy die angelegten Kriterien wesentlich besser erfüllen als die Plattformen auf Basis von LiquidFeedback. Allerdings wurden bei fünf der sechs Plattformen hohe Hürden bereits beim Zugang identifiziert, was die Chance einer Beteiligung über diese Plattformen hemmen kann.

Der folgende Beitrag von Jörg Radtke und Sheree May Saßmannshausen dreht sich ebenfalls um das Thema digitale Bürgerbeteiligung, wobei die technische Seite stärker im Fokus steht. In ihrer Untersuchung eines BürgerInnenbeteiligungsverfah- 
rens in der nordrhein-westfälischen Stadt Olpe gehen sie der Frage nach, welche technischen Optionen eine bessere Rückkopplung zwischen BürgerInnen und Politik erzielen können. Im Ergebnis ihrer Analyse kommen sie zu dem Schluss, dass nicht mehr Instrumente allein, sondern eine Integration von digitalen Instrumenten gekoppelt mit einer stärkeren Rückbindung an politische Prozesse vielversprechend sei, um mehr Nähe zwischen BürgerInnen und politischen Institutionen herzustellen und somit Repräsentationsbeziehungen zu stärken.

Im abschließenden Beitrag der HerausgeberInnen dieses Heftes ${ }^{2}$ unterziehen Isabelle Borucki, Dennis Michels und Stefan Marschall die bestehenden Modelle zur Digitaldemokratie einer kritischen Prüfung und gehen der These nach, dass eine adäquate Theoretisierung und Auseinandersetzung mit der Digitaldemokratie nur funktionieren kann, wenn man ihre Funktionslogiken anerkennt und nicht umgekehrt, die bekannten Konzepte und Formate aus der vordigitalen Zeit anwenden möchte. Sie skizzieren - auch angesichts der aktuellen technischen und gesellschaftlichen Dynamik - welchen Fragen sich die Sozialwissenschaften mit Blick auf die Digitalisierung der Demokratie zukünftig zuwenden sollten und welche methodischen wie auch theoretischen Ansätze dabei vielversprechend sein könnten.

Funding Open Access funding provided by Projekt DEAL.

Open Access Dieser Artikel wird unter der Creative Commons Namensnennung 4.0 International Lizenz veröffentlicht, welche die Nutzung, Vervielfältigung, Bearbeitung, Verbreitung und Wiedergabe in jeglichem Medium und Format erlaubt, sofern Sie den/die ursprünglichen Autor(en) und die Quelle ordnungsgemäß nennen, einen Link zur Creative Commons Lizenz beifügen und angeben, ob Änderungen vorgenommen wurden.

Die in diesem Artikel enthaltenen Bilder und sonstiges Drittmaterial unterliegen ebenfalls der genannten Creative Commons Lizenz, sofern sich aus der Abbildungslegende nichts anderes ergibt. Sofern das betreffende Material nicht unter der genannten Creative Commons Lizenz steht und die betreffende Handlung nicht nach gesetzlichen Vorschriften erlaubt ist, ist für die oben aufgeführten Weiterverwendungen des Materials die Einwilligung des jeweiligen Rechteinhabers einzuholen.

Weitere Details zur Lizenz entnehmen Sie bitte der Lizenzinformation auf http://creativecommons.org/ licenses/by/4.0/deed.de.

\section{Literatur}

Baecker, Dirk. 2018. 4.0 oder Die Lücke die der Rechner lässt. Leipzig: Merve.

Bieber, Christoph (Hrsg.). 2001. ParteiPolitik 2.0. Der Einfluss des Internet auf parteiinterne Kommunikations- und Organisationsprozesse. Studie für die Friedrich-Ebert-Stiftung. Wirtschafts- und Sozialpolitisches Forschungs- und Beratungszentrum. Bonn: Friedrich-Ebert-Stiftung Abt. Wirtschaftspolitik.

Brake, David R. 2014. Are we all online content creators now? Web 2.0 and digital divides. Journal of Computer-Mediated Communication 19(3):591-609.

Crouch, Colin. 2008. Postdemokratie. Frankfurt am Main: Suhrkamp.

Dahlberg, Lincoln. 2011. Re-constructing digital democracy: an outline of four "positions". New Media \& Society 13(6):855-872. https://doi.org/10.1177/1461444810389569.

Dohle, Marco, Olaf Jandura, und Gerhard Vowe. 2014. Politische Kommunikation in der OnlineWelt. Dimensionen des strukturellen Wandels politischer Kommunikation. Zeitschrift für Politik 61(4):414-436.

\footnotetext{
${ }^{2}$ Dieser ist nicht in einem Peer-Review-Prozess begutachtet worden.
} 
Geisler, Alexander, und Ulrich Sarcinelli. 2002. Modernisierung von Wahlkämpfen und Modernisierung von Demokratie? In Wahl-Kämpfe, Hrsg. Andreas Dörner, Ludgera Vogt, 43-65. Frankfurt am Main: Suhrkamp.

Helbing, Dirk. 2019. Towards digital enlightenment: essays on the dark and light sides of the digital revolution. Wiesbaden: Springer.

Henn, Philipp, Olaf Jandura, und Gerhard Vowe. 2016. The traditional paradigm of political communication research reconstructed. In Political communication in the online world. Theoretical approaches and research designs, Hrsg. Gerhard Vowe, Philipp Henn, 11-25. New York, London: Routledge.

Hepp, Andreas, Andreas Breiter, und Uwe Hasebrink. 2018. Rethinking transforming communications: an introduction. In Communicative figurations. Transforming communications in times of deep mediatization, Hrsg. Andreas Hepp, Andreas Breiter, und Uwe Hasebrink, 3-13. Cham: Palgrave Macmillan.

Hofmann, Jeanette. 2019. Mediatisierte Demokratie in Zeiten der Digitalisierung - Eine Forschungsperspektive. In Politik in der digitalen Gesellschaft. Zentrale Problemfelder und Forschungsperspektiven, Hrsg. Jeanette Hofmann, Norbert Kersting, Claudia Ritzi, und Wolf J. Schünemann, 27-45. Bielefeld: transcript.

Hofmann, Jeanette, Norbert Kersting, Claudia Ritzi, und Wolf J. Schünemann (Hrsg.). 2019. Politik in der digitalen Gesellschaft. Zentrale Problemfelder und Forschungsperspektiven. Bielefeld: transcript.

Kersting, Norbert. 2019. Online Partizipation: Evaluation und Entwicklung - Status quo und Zukunft. In Politik in der digitalen Gesellschaft. Zentrale Problemfelder und Forschungsperspektiven, Hrsg. Jeanette Hofmann, Norbert Kersting, Claudia Ritzi, und Wolf J. Schünemann, 105-121. Bielefeld: transcript.

Kneuer, Marianne. 2013. Bereicherung oder Stressfaktor? Überlegungen zur Wirkung des Internets auf die Demokratie. In Das Internet. Bereicherung oder Stressfaktor für die Demokratie?, Hrsg. Marianne Kneuer, 7-31. Baden-Baden: Nomos.

Korte, Karl-Rudolf, und Manuel Fröhlich. 2009. Politik und Regieren in Deutschland. Strukturen, Prozesse, Entscheidungen, 3. Aufl., Paderborn: UTB.

Korte, Karl-Rudolf, Dennis Michels, Jan Schoofs, Niko Switek, und Kristina Weissenbach. 2018. Parteiendemokratie in Bewegung. Organisations- und Entscheidungsmuster der deutschen Parteien im Vergleich. Baden-Baden: Nomos.

Marschall, Stefan. 1998. Parlamentarische Repräsentation in der Informationsgesellschaft. Zeitschrift für Politik 45(3):282-299.

Miller, Michael L., und Cristian Vaccari. 2020. Digital threats to democracy: comparative lessons and possible remedies. International Journal of Press/Politics https://doi.org/10.1177/1940161220922323.

Min, Seong-Jae. 2010. From the digital divide to the democratic divide: Internet skills, political interest, and the second-level digital divide in political Internet use. Journal of Information Technology \& Politics 7(1):22-35.

Nassehi, Armin. 2019. Muster. Theorie der digitalen Gesellschaft, 3. Aufl., München: C.H.Beck.

Rheingold, Howard. 1993. Virtual community: homesteading on the electronic frontier. Reading: AddisonWesley.

Saxer, Ulrich. 1998. System, Systemwandel und politische Kommunikation. In Politische Kommunikation in der demokratischen Gesellschaft. Ein Handbuch mit Lexikonteil, Hrsg. Ottfried Jarren, 21-64. Opladen: Westdeutscher Verlag.

Schröder, Michael, und Axel Schwanebeck (Hrsg.). 2017. Big Data - In den Fängen der Datenkraken. Baden-Baden: Nomos. https://doi.org/10.5771/9783845283678.

Stalder, Felix. 2016. Kultur der Digitalität. Berlin: Suhrkamp.

Thaa, Winfried, und Christian Volk (Hrsg.). 2018. Formwandel der Demokratie. Baden-Baden: Nomos. https://doi.org/10.5771/9783845295787.

Tucker, Joshua A., Yannis Theocharis, Margaret E. Roberts, und Pablo Barberá. 2017. From liberation to turmoil: social media and democracy. Journal of Democracy 28(4):46-59.

Wei, Lu. 2012. Number matters. The multimodality of Internet use as an indicator of the digital inequalities. Journal of Computer-Mediated Communication 17(3):303-318.

Weiß, Ulrich. 1998. Das Politische am Internet. Eine politikphilosophische Reflexion. In Internet Und Demokratie, Hrsg. Winand Gellner, Fritz von Korff, 27-42. Baden-Baden: Nomos. 